\title{
Changes in mediators of inflammation and pro-thrombosis after 12 months of dietary modification in adults with metabolic syndrome.
}

\author{
SK Rahamon ${ }^{1}$, UA Fabian ${ }^{1}$, MA Charles-Davies ${ }^{1}$, JA Olaniyi ${ }^{2}$, AA Fasanmade ${ }^{3}$, KS Akinlade ${ }^{1}$,

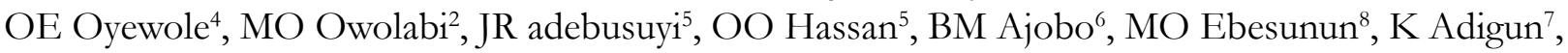 \\ OO Popoola ${ }^{1}$, W Omiyale ${ }^{1}$, OG Arinola ${ }^{1}$, EO Agbedana ${ }^{1}$.
}

1. Department of Chemical Pathology, College of Medicine, University of Ibadan, Ibadan, Nigeria

2. Department of Haematology, College of Medicine, University of Ibadan, Ibadan, Nigeria.

3. Department of Medicine, College of Medicine, University of Ibadan, Ibadan, Nigeria

4. Department of Health Promotion and Education, College of Medicine, University of Ibadan, Ibadan, Nigeria

5. Department of Medical Social Services, University College Hospital, Ibadan, Nigeria

6. Dietetics Department, University College Hospital, Ibadan, Nigeria

7. Department of Family Medicine, University College Hospital, Ibadan, Nigeria

8. Department of Chemical Pathology, College of Health Sciences, Olabisi Onabanjo University, Ago-Iwoye, Nigeria

\begin{abstract}
Objective: This study evaluated the effects of a 12-month dietary modification on indices of inflammation and pro-thrombosis in adults with metabolic syndrome (MS).

Materials and methods: This longitudinal study involved 252 adults with MS recruited from the Bodija market, Ibadan and its environs. Participants were placed on 20\%,30\% and 50\% calories obtained from protein, total fat and carbohydrate respectively and were followed up monthly for 12 months. Anthropometry and blood pressure were measured using standard methods. Fasting plasma glucose (FPG), total cholesterol (TC), triglycerides (TG), high density lipoprotein-cholesterol (HDL-C), fibrinogen, plasminogen activator inhibitor-1 (PAI-1)], interleukin-6 (IL-6) and interleukin-10 (IL-10) were measured using spectrophotometric methods and ELISA as appropriate. Data was analysed using ANCOVA, Student's t-test, Mann-Whitney U and Wilcoxon signed-rank tests. P-values less than 0.05 were considered significant.

Results: After 6 months of dietary modification, there was a significant reduction in waist circumference (WC), while the levels of HDL-C, fibrinogen and PAI-1 were significantly increased when compared with the corresponding baseline values. However, WC and fibrinogen reduced significantly, while HDL-C and IL-10 significantly increased after 12 months of dietary modification as compared with the respective baseline values.

Conclusion: Long-term regular dietary modification may be beneficial in ameliorating inflammation and pro-thrombosis in metabolic syndrome.
\end{abstract}

Keywords: Dietary modification, fibrinogen, interleukins, metabolic syndrome, plasminogen activator inhibitor.

DOI: https://dx.doi.org/10.4314/ahs.v17i2.20

Cite as: Rahamon SK, Fabian UA, Charles-Davies MA, Olaniyi JA, Fasanmade AA, Akinlade KS, Oyewole OE, Owolabi MO, adebusuyi JR, Hassan OO, Ajobo BM, Ebesunun MO, Adigun K, Popoola OO, Omiyale W, Arinola OG, Agbedana EO. Changes in mediators of inflammation and prothrombosis after 12 months of dietary modification in adults with metabolic syndrome. Afri Health Sci. 2017;17(2): 453-462. https:/ / dx.doi.org/10.4314/abs.v17i2.20

\section{Corresponding author:}

OG Arinola,

Immunology Unit,

Department of Chemical Pathology,

College of Medicine,

University of Ibadan 200212,

Nigeria.

Mobile: +2348023451520

Email: drarinolaog64@yahoo.com

\section{Introduction}

Metabolic syndrome (MS) is a constellation of central obesity, insulin resistance, hyperglycaemia, hypertension, dyslipidaemia and other interconnected factors which increase the risk of cardiovascular diseases (CVDs) and type 2 diabetes mellitus (T2DM) $)^{1,2}$.

The prevalence of MS continues to rise due to the rapidly growing number of inactive people, obesity, increased urbanization, industrialization and mechanization ${ }^{3,4}$. Depending on the country, approximately 1 adult in 4 (25\%) is believed to have MS ${ }^{5}$. Reports from the United States 
showed that the prevalence of MS among US adults is about $40 \%{ }^{6,7}$. A similar prevalence $(30 \%)$ was reported by Mannuci et al. ${ }^{8}$ in Italy. However MS, which is attributed to a positive energy balance, is now prevalent in developing countries where poverty and malnutrition are problems. In Cameroon, Fezeu et al. ${ }^{9}$ reported a prevalence of $5.9 \%$ while Motala et al. ${ }^{10}$ and Erasmus et al. ${ }^{11}$ reported prevalence rates of $26.5 \%$ and $60.60 \%$ in Black and Coloured communities of South Africa respectively. Also, the prevalence of MS appears to be rising among Nigerians. ${ }^{12}$ In 2010, Adegoke et al. ${ }^{13}$ reported a prevalence of $12.1 \%$ in a rural community in Nigeria while Ghazali and Sanusi ${ }^{14}$ and Charles-Davies et al. ${ }^{15}$ reported a prevalence of $36.7 \%$ and $33.1 \%$ respectively among adults living in Ibadan, a Nigerian city. These reports clearly indicate that CVDs and T2DM might grow in epidemic proportion in the nearest future in Nigeria unless, urgent and focused clinical interventions are made.

Chronic inflammation and pro-thrombosis are important components of MS and they play significant roles in its pathophysiology ${ }^{16-20}$. However, the MS-associated inflammation does not resemble the classical inflammation. It is considered as an aberrant form of immune response that is triggered by nutrients, and thus referred to as meta-inflammation or para-inflammation ${ }^{21,22}$.

Therapeutic lifestyle changes (diet and exercise), pharmacologic therapies and surgery have been recommended for the management of $\mathrm{MS}^{23-25}$. However, due to challenges faced by the pharmacologic therapies and surgery especially, in developing countries where poverty and access to quality healthcare are problems; it becomes clear that change of lifestyle such as dietary modification could be a viable therapeutic approach in managing MS. Roberts et al. ${ }^{26}$ and Akbaraly et al. ${ }^{27}$ reported significant changes in metabolic risk factors in individuals with MS following short-term and long-term dietary modifications. A preliminary study in our laborator $y^{28}$ indicated a positive impact of short term dietary modification on indices of inflammation, oxidative stress as well as cardiovascular risks.

To provide information that may be useful in designing a nutritional intervention to prevent the development of MS as well as in the management of MS with a view of preventing possible progression to T2DM, this study determined the possible attenuating effects of Nigerian diets, which are predominantly starchy foods, on selected inflammatory and pro-thrombosis factors in adults with MS after 12 months of dietary modification.

\section{Materials and methods Subjects}

A total of 252 apparently healthy adults with MS were recruited into this prospective study. The participants were cohorts involved in a study titled "Risk Assessment of Type 2 Diabetes Mellitus and Dementia in Nigerians with Metabolic Syndrome"29. Briefly, 790 apparently healthy participants were consecutively enrolled into the cohortstudy and were screened for MS using the International Diabetes Federation diagnostic criteria ${ }^{30}$. Two hundred and fifty two (252) of them fulfilled the criteria for MS and were thus, selected for this prospective study. Participants who were underweight, pregnant women, those with HIV/hepatitis infection and those on anti-inflammatory and pro-fibrinolysis agents were excluded from the study.

To further establish the metabolic, inflammatory and prothrombotic alterations in MS, 40 adults who did not have any component of MS were purposively selected from amongst the cohorts as controls.

\section{Ethical consideration}

The study was carried out after an ethical approval from the University of Ibadan/University College Hospital (UI/UCH) Joint Ethics Review Committee and written informed consent was obtained from each participant.

\section{Dietary prescription}

All the participants with MS received a comprehensive consultation with a principal Dietitian in the Department of Dietetics, University College Hospital, Ibadan, Nigeria. The need for behavioural change such as dietary modification was discussed with all the participants as one of the ways to enhance good health. Different cooking methods were also discussed with all the participants. Thereafter, individual dietary intake was assessed using a 24 - hour dietary recall. The diets were documented by the Dietitian, and to obtain precise information, each subject was asked if that was their typical pattern of intake, how often they ate per day, what variety of food they consumed, how the food was prepared, what the serving size was, and the brand of food/meal they usually consumed. Based on the 
information provided, total caloric intake obtained from protein, total fat, and carbohydrate was calculated and pegged at $20 \%, 30 \%$ (including $14 \%$ polyunsaturated fat) and $50 \%$ respectively. Each participant was provided with verbal and written instructions containing list of recipes and quantities of different food to be consumed to meet the desired dietary requirement.

Each participant with MS was seen monthly for 12 months by the Dietitian and information on compliance was obtained. In addition, assessment of compliance was done by calculating waist-to-height ratio (WHtR) at 6 and 12 months. Martínez-González et al. ${ }^{31}$ reported an inverse association between adherence to the Mediterranean diet and WHtR than BMI and WC.

\section{Sample collection}

After an overnight fast, $15 \mathrm{mls}$ of venous blood were collected from of each participant at baseline (before the commencement of dietary modification), 6 months and 12 months (post-dietary modification). Samples were collected only once (at the beginning of the study) in the controls. Prior to the scheduled 6 and 12 months visits, reminder telephone calls were made to each participant with MS. Samples were dispensed into fluoride oxalate bottles, EDTA-containing sample bottles, citrate-containing sample bottles and plain sample bottles to obtain plasma and serum as appropriate. Serum and plasma samples obtained were stored at $-20^{\circ} \mathrm{C}$ until analyzed.

\section{Anthropometric and blood pressure measurements}

Body weight, height, body mass index (BMI), waist circumference (WC), hip circumference (HC), waist-hip ratio (WHR), waist-height ratio (WHtR), \% body fat (BF), and blood pressure (BP) were obtained from the participants by standard methods as described elsewhere ${ }^{29}$.

\section{Fasting plasma glucose and lipids}

Enzymatic methods were used for the determination of plasma TG, TC, HDL-C and FPG, while LDL-C was calculated using Frieldwald et al. formula ${ }^{32}$.

\section{Inflammatory and pro-thrombotic factors}

Serum levels of inflammatory cytokines [interleukin-6 (IL-6) and interleukin-10 (IL-10)] were determined using ELISA (Boster Biological Technology Co., Inc, USA). Similarly, serum levels of pro-thromobotic factors [human plasminogen activator inhibitor-1 (PAI-1) and fibrinogen] were determined using ELISA (Assaypro LLC, USA).

\section{Statistical analysis}

Statistical analysis was carried out using SPSS statistical software version 17.0 for windows. The Gaussian distribution of all the quantitative variables was assessed using histogram with normal curve. Values were reported as mean \pm standard deviation or median (interquartile range) for normally and non-normally distributed data respectively. At baseline, groups were compared using unpaired Student's t-test and Mann-Whitney U depending on the distribution of the variables. Age and gender were adjusted for, using Analysis of Covariance (ANCOVA) when comparing the MS group with control. Comparing the baseline and post-intervention data, the differences in means/medians were determined using paired Student's t-test or Wilcoxon signed-rank test as appropriate. P-values less than 0.05 were considered statistically significant.

\section{Results}

At the end of the 12 months of dietary modification, there was a high drop-out rate $(71.43 \%$ at 6 -months and $85.72 \%$ at 12 -months) in participants with MS. Only 32 participants with MS had complete baseline, 6 months and 12 months data hence, were compared with the controls.

As shown in Table 1, body weight, BMI, WC, HC, WHR, WHtR, \% body fat, systolic and diastolic BP, FPG, fibrinogen and PAI-1 were significantly higher while HDL-C and IL-10 were significantly lower in participants with MS compared with the corresponding control values. However, after adjusting for age and gender, only the mean HDL-C was significantly lower, while body weight, BMI, WC, HC, waist-hip ratio, WHtR, \% body fat, fibrinogen, systolic and diastolic BP were still higher in participants with MS compared with the respective values in the controls. 
Table 1: Gender, anthropometric, clinical, metabolic, inflammatory and prothrombosis indices in participants with MS and controls

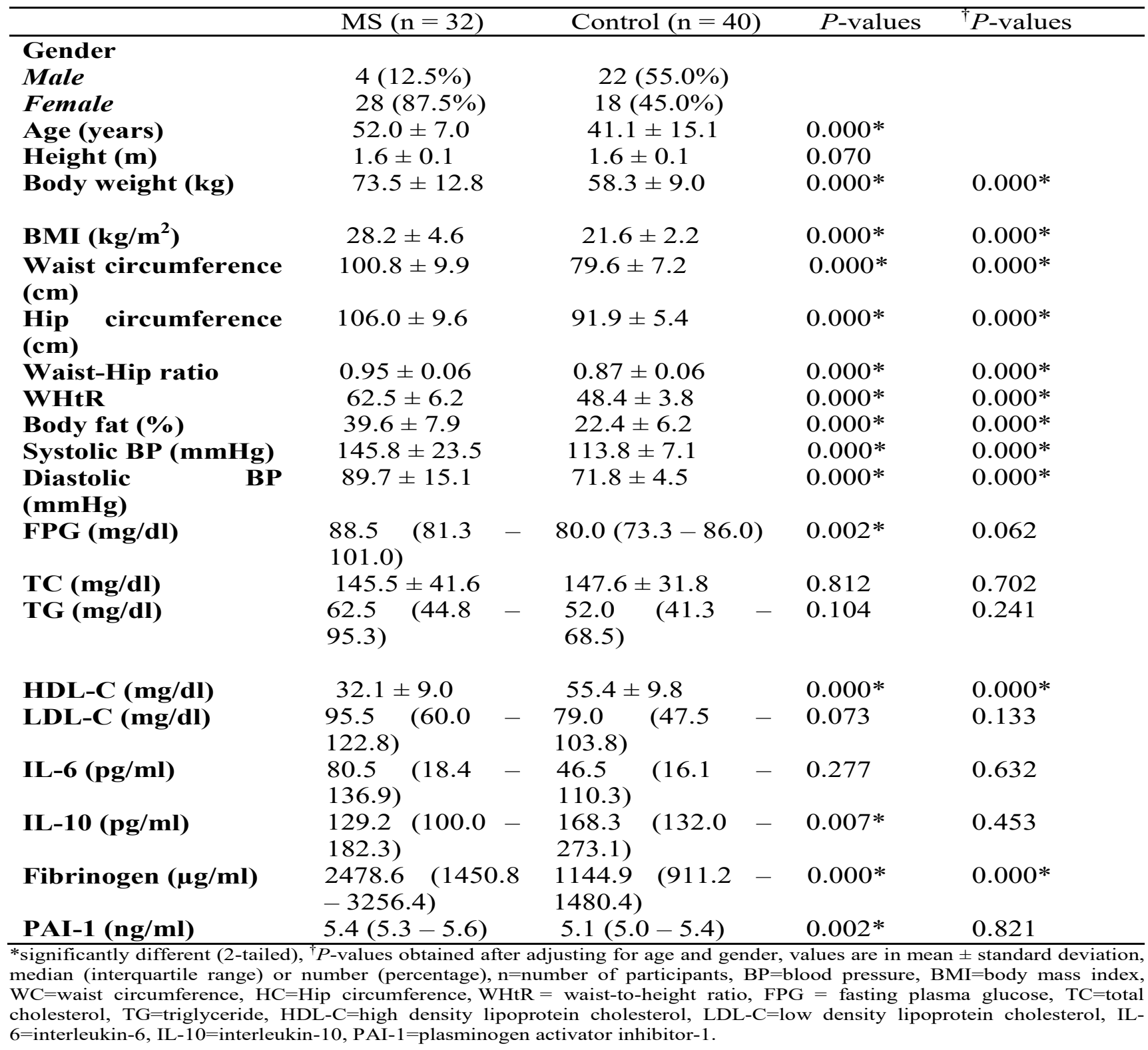

At the end of 12 months of dietary modification, the mean body weight, BMI, WC, HC, WHR, WHtR, \% body fat, SBP and DBP progressively reduced, while the HDL-C progressively increased from baseline through 12 months (Table 2).

Comparing baseline and 6-months, there were significant reductions in body weight, WC, WHtR, \% body fat, SBP and DBP while HDL-C, TC, fibrinogen and PAI-1 had significant elevations in the MS subjects (Table 2). Similarly, there were significant reductions in body weight, BMI, SBP, DBP, WC, HC, WHR, WHtR, \% body fat and fibrinogen while HDL-C and IL-10 had significant elevations at 12 -months compared with the respective baseline. However, only the body weight, BMI, WC, waist-hip ratio, WHtR, fibrinogen and PAI-1 had significant reductions at 12 -months compared with the respective values at 6-months (Table 2). 


\section{Table 2: Anthropometric, clinical, metabolic, inflammatory and prothrombosis indices at baseline, 6 months and 12 months}

\begin{tabular}{|c|c|c|c|}
\hline & Baseline $(n=32)$ & 6months $(n=32)$ & 12 months $(n=32)$ \\
\hline$\overline{\text { Body weight }(\mathrm{kg})}$ & $73.5 \pm 12.8$ & $71.3 \pm 12.2^{\mathrm{a} \uparrow}$ & $69.5 \pm 11.7^{\mathrm{a}^{\dagger}, \mathrm{b} \dagger}$ \\
\hline $\operatorname{BMI}\left(\mathrm{kg} / \mathrm{m}^{2}\right)$ & $28.2 \pm 4.6$ & $27.6 \pm 4.4$ & $26.8 \pm 4.0^{\mathrm{a} \dagger, \mathrm{b} \dagger}$ \\
\hline Waist circumference $(\mathrm{cm})$ & $100.8 \pm 9.9$ & $97.9 \pm 8.6^{\mathrm{a}^{\dagger}}$ & $94.3 \pm 9.2^{\mathrm{a} \dagger, \mathrm{b} \dagger}$ \\
\hline Hip circumference (cm) & $106.0 \pm 9.6$ & $104.3 \pm 9.3$ & $102.4 \pm 8.5^{\mathrm{a} \dagger}$ \\
\hline Waist-Hip ratio & $0.95 \pm 0.06$ & $0.94 \pm 0.06$ & $0.92 \pm 0.07^{\mathrm{a}^{\dagger}, \mathrm{b} \dagger}$ \\
\hline WHtR & $62.8 \pm 6.0$ & $61.1 \pm 5.5^{\mathrm{a}^{\dagger}}$ & $58.8 \pm 5.3^{\mathrm{a}^{\dagger \dagger} \mathrm{b}_{\dagger}^{\dagger}}$ \\
\hline Body fat (\%) & $39.6 \pm 7.9$ & $38.2 \pm 8.2^{\mathrm{a}^{\dagger}}$ & $36.4 \pm 8.2^{\mathrm{a \dagger}}$ \\
\hline Systolic BP (mmHg) & $145.8 \pm 23.5$ & $132.6 \pm 19.0^{\mathrm{a} \dagger}$ & $130.3 \pm 17.9^{\mathrm{a} \dagger}$ \\
\hline Diastolic BP (mmHg) & $89.7 \pm 15.1$ & $85.2 \pm 12.9^{\mathrm{a}^{\dagger}}$ & $82.8 \pm 11.1^{\mathrm{a}^{\dagger}}$ \\
\hline FPG $(\mathrm{mg} / \mathrm{dl})$ & $88.5(81.3-101.0)$ & $90.0(82.0-98.50)$ & $90.5(79.3-96.8)$ \\
\hline $\mathrm{TC}(\mathrm{mg} / \mathrm{dl})$ & $145.5 \pm 41.6$ & $165.6 \pm 40.7^{\mathrm{a}:}$ & $154.9 \pm 45.0$ \\
\hline $\mathrm{TG}(\mathrm{mg} / \mathrm{dl})$ & $62.5(44.8-95.3)$ & $72.0(52.0-86.5)$ & $80.5(59.3-103.3)$ \\
\hline HDL-C (mg/dl) & $32.1 \pm 9.0$ & $48.8 \pm 16.8^{\mathrm{a} \ddagger}$ & $52.1 \pm 13.2^{\mathrm{a} \ddagger}$ \\
\hline LDL-C (mg/dl) & $95.5(60.0-122.8)$ & $102.0(72.0-119.0)$ & $88.0(59.0-115.0)$ \\
\hline IL-6 (pg/ml) & $80.5(18.4-136.9)$ & $85.8(40.0-245.1)$ & $111.2(41.6-181.8)$ \\
\hline IL-10 (pg/ml) & $129.2(100.0-182.3)$ & $126.5(81.8-201.8)$ & $140.4(94.4-192.1)^{\mathrm{a}:}$ \\
\hline Fibrinogen $(\mu \mathrm{g} / \mathrm{ml})$ & $2478.6(1450.8-3256.4)$ & $3302.9(2247.0-4138.9)^{\mathrm{a} a}$ & 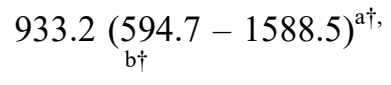 \\
\hline PAI-1 (ng/ml) & $5.4(5.3-5.6)$ & $5.5(5.4-5.6)^{\mathrm{a} .5}$ & $5.4(5.4-5.5)^{\mathrm{b} \dagger}$ \\
\hline
\end{tabular}

*significantly different (2-tailed), values are in mean \pm standard deviation or median (interquartile range), $\mathrm{n}=$ number of participants ${ }^{\mathrm{a} \dagger}$ significantly reduced when compared with baseline, ${ }^{\mathrm{a} \ddagger}$ significantly increasedcompared with baseline, ${ }^{\mathrm{b} \dagger}$ significantly reduced compared with 6-month, ${ }^{b *}$ significantly increased compared with 6-month, $\mathrm{BP}=$ blood pressure, $\mathrm{BMI}=$ body mass index, $\mathrm{WC}=$ waist circumference, $\mathrm{HC}=\mathrm{Hip}$ circumference, $\mathrm{WHtR}=$ waist-to-height ratio, $\mathrm{FPG}=$ fasting plasma glucose, $\mathrm{TC}=$ total cholesterol, TG=triglyceride, HDL-C=high density lipoprotein cholesterol, LDL-C=low density lipoprotein cholesterol, IL6=interleukin-6, IL-10=interleukin-10, PAI-1=plasminogen activator inhibitor-1.

\section{Discussion}

Type 2 diabetes mellitus and cardiovascular diseases epidemic is on the rise due to increasing prevalence of metabolic syndrome (MS) and obesity. Although MS is considered an energy excess health problem, it is now prevalent in developing countries where poverty and malnutrition are common.

The observed significant elevation of anthropometric indices, blood pressure and FPG, and reduced HDL-C levels in participants with MS compared with controls support earlier reports ${ }^{14,33}$. This has been attributed to an imbalance between energy expenditure and storage, insulin resistance and activation of renin-angiotensin-aldosterone system (RAAS) among others. Our observation, together with earlier reports, further confirm the important role played by these indices in the development as well as harmonization of various diagnostic criteria of MS.

Individuals with MS typically manifest pro-thrombic and pro-inflammation states. PAI-1 has been shown to be a good predictor of MS $^{34,35}$. High plasma PAI-1 has been associated with thrombus formation which causes cardiovascular events ${ }^{36}$. The elevated level of PAI-1 observed in participants with MS compared with the controls is in line with the report of Juhan-Vague et al. ${ }^{37}$. Our observation might indicate increased PAI-1 production from the rapidly expanding adipocytes and/or macrophages infiltrating the adipose tissue. Dellas and Loskutoff ${ }^{38}$ and Alessi and Juhan-Vague ${ }^{39}$ reported that hepatocytes, endothelial cells, adipocytes, megakaryocytes, and macrophages infiltrating the adipose tissue synthesize and secrete PAI-1 into the circulation. Furthermore, fat redistribution phenotype, insulin resistance, cortisol production, glucidolipidic disturbances, renin angiotensin system and oxidative stress have also been identified as possible inducers of PAI-1 synthesis in MS ${ }^{39}$.

Fibrinogen is an acute phase reactant that is increased in inflammatory states. It is an independent risk factor for cardiovascular diseases ${ }^{40}$. Our observed elevated level in participants with MS compared with the controls corroborates the report of Ford ${ }^{41}$. This observation could be 
attributed to the MS-associated inflammation resulting from myriads of inducers. For example, Toll-like receptor-4 (TLR4) ligands such as saturated fatty acids and oxidative stress have been shown to activate NF-kB and activator protein-1 transcription factors leading to enhanced pro-inflammation in $\mathrm{MS}^{42}$.

Central obesity has been shown to induce phenotypic switch from alternatively activated macrophages (M2) to classically activated macrophages (M1) which is characterized by reduction in anti-inflammation and an increase in pro-inflammation ${ }^{43}$. The observed lower level of IL-10 in participants with MS compared with the controls is in line with the report of Esposito et al. ${ }^{44}$. Our observation could be as a result of shift in adipose tissue macrophages (ATM) population. Studies have shown that there is reduced visceral adipose tissue (VAT) resident regulatory $\mathrm{T}$ cells (Tregs), increased proportion of Th1 T cells and $\mathrm{CD}^{+} \mathrm{T}$ cells, and increased ratio of $\mathrm{Th} 1$ and $\mathrm{Th} 17$ to Treg in $\mathrm{MS}^{45,46}$.

After adjusting for age and gender using multivariate analysis however, body weight, BMI, WC, HC, waist-hip ratio, \% body fat, fibrinogen, SBP and DBP were still elevated, while the HDL-C was still reduced in participants with MS compared with the respective values in the control group. This observation probably suggests that these aforementioned parameters are indeed, features of MS irrespective of age and gender. van Exel et al. ${ }^{47}$ reported that women have lower IL-10 and TNF- $\alpha$ production capacity than men. This might explain our observed insignificant differences in IL-10 levels after adjusting for gender since majority of the participants with MS were females.

Adoption of a healthier lifestyle has been identified as an important step in reducing the excess cardiovascular risk associated with $\mathrm{MS}^{25}$. The observed progressive reduction in all the anthropometric indices (body weight, BMI, WC,HC, waist-hip ratio, WHtR, \% body fat) and blood pressure readings (SBP and $\mathrm{DBP}$ ), and the concomitant rise in HDL-C concentration from baseline through 12-month HDL-C level corroborates the reports of Roberts et al. ${ }^{26}$ and Akbaraly et al. ${ }^{27}$ who observed significant beneficial changes in the cardio-metabolic risk factors in MS subjects following short-term and long-term dietary modification. Our observation probably indicates that diet modification results in less energy storage and increased utilization of stored energy thereby, improving the cardio-metabolic risk factors as observed. Although verbal report and recall methods were used to assess dietary compliance, the consistent observed progressive reduction in WHtR probably indicates an appreciable adherence to the dietary modification as it has been report$\mathrm{ed}^{31}$ that WHtR has an inverse association with adherence to the Mediterranean diet.

Adipocyte hypertrophy facilitates cell rupture and evokes an inflammatory reaction which has been associated with increased cardiovascular risks and other metabolic disturbances $^{33,48,49}$. IL-10 is an effective anti-inflammatory cytokine which regulates the inflammatory response ${ }^{47}$. In this study, IL-10 did not increase at 6-months but increased at 12-months compared with baseline. Its elevation at 12 months could indicate that there is a reversal in the induction of phenotypic switch from alternatively activated macrophages (M2) to classically activated macrophages (M1) which signifies reduced infiltration of the adipose tissue by macrophages favouring anti-inflammation ${ }^{43}$. Macrophages form a crown-like structure around necrotic/adipocyte debris which indicates that their presence in adipose tissue is predominantly for clearance purpos$\mathrm{es}^{50}$. For each dead adipocyte, several macrophages are recruited which amplifies the inflammatory response ${ }^{51}$. After this, the macrophages phagocytose the cell debris of the apoptotic cells and engage in an anti-inflammatory programme $e^{52,53}$. Also, the observation in this study could be an indication that there is increased VAT resident regulatory T cells (Tregs), reduced proportion of Th1 T cells and $\mathrm{CD}^{+} \mathrm{T}$ cells, and reduced ratio of $\mathrm{Th} 1$ and Th17 to Treg after dietary modification.

The observed reduction in fibrinogen level at 12-months compared with baseline and 6-months supports the report of Ditschuneit et al. ${ }^{54}$ who reported a fall in plasma fibrinogen level accompanying a reduction in BMI after a 6-month low calorie diet intake. Our observation could be attributed to reduction in MS associated inflammation post-dietary modification. The mechanism for the observed lower fibrinogen level and higher IL-10 level post-dietary modification could be explained through the reduction in central adiposity. Reduction in WC (as observed) brought about by the dietary modification reduced infiltration of the adipose tissue by macrophages and/or reduced phenotypic switch from alternatively ac- 
tivated macrophages (M2) to classically activated macrophages (M1) thereby culminating in reduced pro-inflammation and increased anti-inflammation.

Increased PAI-1 level has been shown to be a true component of $\mathrm{MS}^{39,55}$. The median PAI-1 level that was significantly higher at 6-months reduced significantly at 12-months, paralleling the baseline value. These non-specific changes contradict the report of Folsom et al. ${ }^{56}$ who reported a decrease in plasma PAI-1 level following hypocaloric diet. Our observation could not be explained at the moment but the observed inconsistent changes in PAI-1 levels might indicate that PAI-1 changes in MS do not follow the usual pattern of inflammation and dyslipidaemia. Rega et al. ${ }^{57}$ reported that increased expression of PAI-1 in MS is not associated with IL-6 driven inflammation and dyslipidaemia.

The high drop-out rate observed in this study was a major limitation. This observation however, highlighted why early diagnosis and prompt management of MS and its associated diseases could be difficult as majority of adult Nigerians especially, those with no formal education are not easily convinced that there is the need to go for routine medical check-up even, when they feel apparently healthy.

\section{Conclusion}

This study shows that six and twelve months of dietary modification effectively reversed the conventional components of MS. However, the changes in mediators of inflammation and pro-thrombosis were only prominent after 12 months of dietary modification. Therefore, long-term regular dietary modification may be a useful tool in improving inflammation and pro-thrombosis in metabolic syndrome.

\section{Conflicts of interest}

Authors have no competing interests to declare.

\section{Acknowledgement}

This research work was partly funded by the 2009 Multidisciplinary Research Grant of the University of Ibadan MacArthur Foundation and the University of Ibadan Senate Research Grant (SRG/COM/2010/2C).

\section{References}

1. Alberti KG, Eckel RH, Grundy SM, Zimmet PZ, Cl- eeman JI, et al. Harmonizing the metabolic syndrome: a joint interim statement of the International Diabetes Federation Task Force on Epidemiology and Prevention; National Heart, Lung, and Blood Institute; American Heart Association; World Heart Federation; International Atherosclerosis Society; and International Association for the Study of Obesity. Circulation. 2009; 120: 16401645 PubMed.

2. Kassi E, Pervanidou P, Kaltsas G, Chrousos G. Metabolic syndrome: definitions and controversies. BMC Medicine. 2011; 9: 48.

3. Gaziano TA, Galea G, Reddy KS. Scaling up interventions for chronic disease prevention: the evidence. Lancet. 2007; 370: 1939-1946.

4. Hollman G, Kristenson M. The prevalence of the metabolic syndrome and its risk factors in a middle-aged Swedish population--mainly a function of overweight? European Journal of Cardiovascular Nursing. 2008; 7(1): 21 26.

5. Hossain P, Kawar B, El Nahas M. Obesity and diabetes in the developing world- a growing challenge. New England Journal of Medicine. 2007; 356: 213-215.

6. Ford ES. Prevalence of the Metabolic Syndrome Defined by the International Diabetes Federation Among Adults in the U.S. Diabetes Care. 2005; 28(11): 2745-2749 7. Li C, Ford ES, McGuire LC, Mokdad AH. Increasing trends in waist circumference and abdominal obesity among US adults. Obesity (Silver Spring). 2007; 15: 216224.

8. Mannucci E, Monami M, Rotella CM. How many components for the metabolic syndrome? Results of exploratory factor analysis in the FIBAR study. Nutrition, Metabolism and Cardiovascular Diseases. 2007; 17(10): 719-726. 9. Fezeu L, Balkau B, Kengne A, Sobngwi E, Mbanya JC. Metabolic syndrome in a Sub-Saharan African setting: Central obesity may be the key determinant. Atherosclerosis. 2007; 193: 70-76.

10. Motala AA, Esterhuizen T, Pirie FJ, Omar MA. The prevalence of metabolic syndrome and determination of the optimal waist circumference cutoff points in a rural South African community. Diabetes Care. 2011; 34(4): 1032-1037.

11. Erasmus RT, Soita DJ, Hassan MS, Blanco-Blanco E, Vergotine $Z$ et al. High prevalence of diabetes mellitus and metabolic syndrome in a South African coloured population: baseline data of a study in Bellville, Cape Town. South African Medical Journal. 2012; 102(11Pt1): 841844. 
12. Bakari AG, Onyemelukwe GC, Sani BG, Aliyu IS, Hassan SS et al. Obesity, overweight and underweight in suburban Northern Nigeria. International Journal of Diabetes and Metabolism. 2007; 15: 68-69.

13. Adegoke OA, Adedoyin RA, Balogun MO, Adebayo RA, Bisiriyu LA et al. Prevalence of metabolic syndrome in a rural community in Nigeria. Metabolic Syndrome and Related Disorders. 2010; 8(1): 59-62.

14. Ghazali SM, Sanusi RA. Waist circumference, waist to hip ratio, and body mass index in the diagnosis of metabolic syndrome in Nigerian subjects. Nigerian Journal of Physiological Sciences. 2010; 25(2): 187-195.

15. Charles-Davies MA, Fasanmade AA, Olaniyi JA, Oyewole OE, Owolabi MO, et al. Prevalent Components of Metabolic Syndrome and their Correlates in Apparently Healthy Individuals in Sub-Saharan Africa. International Journal of Tropical Disease \& Health. 2014; 4(2):740752. PubMed

16. Festa A, Williams K, TracyRP, Wagenknecht LE, Haffner SM. Progression of plasminogen activator inhibitor-1 and fibrinogen levels in relation to incident type 2 diabetes. Circulation. 2006; 113(14): 1753-1759.

17. Hotamisligil GS, Erbay E. Nutrient sensing and inflammation in metabolic diseases. Nature Reviews Immunology. 2008; 8(12): 923-934.

18. Chawla A, Nguyen KD, Sharon Goh YP. Macrophage-mediated inflammation in metabolic disease. Nature Reviews Immunology. 2011; 11: 738-749. Hotamisligil GS. Inflammation and metabolic disorders. Nature. 2006; 444(14): 860-867. PubMed

19. Kalupahana NS, Moustaid-Moussa N, Claycombe KJ. Immunity as a link between obesity and insulin resistance. Molecular Aspects of Medicine. 2012; 33(1): 26-34.

20. Lumeng CN, Saltiel AR. Inflammatory links between obesity and metabolic disease. Journal of Clinical Investigation. 2011; 121(6): 2111-21117

21. Hotamisligil GS. Inflammation and metabolic disorders. Nature. 2006; 444(14): 860 PubMed -867.

22. Medzhitov R. Origin and physiological roles of inflammation. Nature. 2008; 454(7203): 428-435.

23. Longo-Mbenza B, Mvindu HN, On'kin JBK, Bikuku $\mathrm{N}$, Phanzu BK, et al. The deleterious effects of physical inactivity on elements of insulin resistance and metabolic syndrome in Central Africans at high cardiovascular risk. Diabetes and Metabolic syndrome: Clinical Research and Reviews. 2011; 5: 1-6.

24. Matfin G. Challenges in developing therapies for the metabolic syndrome. British Journal of Diabetes and Vascular Disease. 2008; 7: 152.

25. Singh U, Devaraj S, Jialal I. NutritionalModulation of Inflammation in Metabolic Syndrome in Oxidative Stress and inflammatory mechanism in obesity, diabetes and metabolic syndrome eds. Packer L and Sies H. CRS Press pp 227- 242; 2009.

26. Roberts CK, Won D, Pruthi S, Kurtovic S, Sindhu RK et al. Effect of diet and exercise intervention on oxidative stress, inflammation, MMP-9, and monocyte chemotactic activity in men with metabolic syndrome. Journal of Applied Physiology. 2006; 100: 1657-1665.

27. Akbaraly TN, Singh-Manoux A, Tabak AG, Jokela M, Virtanen M et al. Overall diet history and reversibility of the metabolic syndrome over 5 years: the Whitehall II prospective cohort study. Diabetes Care. 2010; 33(11): 2339-2341.

28. Rahamon SK, Charles-Davies MA, Akinlade KS, Olaniyi JA, Fasanmade AA et al. Impact of dietary intervention on selected biochemical indices of inflammation and oxidative stress in Nigerians with metabolic syndrome. European Journal of Nutrition \& Food Safety. 2014; 4(2): $137-$ 149. PubMed

29. Charles-Davies MA, Arinola OG, Fasanmade AA, Olaniyi JA, Oyewole OE et al.Indices of metabolic syndrome in 534 apparently healthy traders in a local market in Ibadan, Nigeria. Journal of US-China Medical Science. 2012; 9(2): 91-100.

30. International Diabetes Federation consensus worldwide de $\neg$ finition of the metabolic syndrome (2006) Available at http://www.idf.org/webdata/docs/IDF_ Meta_def_final.pdf

31. Martínez-González MA, García-Arellano A, Toledo E, Salas-Salvadó J, Buil-Cosiales P, et al. A 14-item Mediterranean diet assessment tool and obesity indexes among high-risk subjects: the PREDIMED trial. PLoS One. 2012; 7(8): e43134.

32. Friedewald WT, Levy RI, Fredricson DS. Estimation of the concentration of low density lipoprotein cholesterol in plasma without use of pre-preparation ultra centrifuge. Clinical Chemistry. 1972; 18: 499-502.

33. Kanbak G, Akalin A, Ozcelik ADE, Bal C. Cardiovascular risk assessment in patients with type 2 diabetes mellitus and metabolic syndrome: Role of biomarkers. Diabetes and Metabolic Syndrome: Clinical Research and Reviews. 2011; 5(1): 7-11

34. Sakkinen PA, Wahl P, Cushman M, Lewis MR, Tra- 
cy RP. Clustering of procoagulation, inflammation, and fibrinolysis variables with metabolic factors in insulin resistance syndrome. American Journal of Epidemiology. 2000; 152(10): 897-907.

35. Anand SS, Yi Q, Gerstein H, Lonn E, Jacobs R et al. Study of Health Assessment and Risk in Ethnic Groups; Study of Health Assessment and Risk Evaluation in Aboriginal Peoples Investigators. Relationship of metabolic syndrome and fibrinolytic dysfunction to cardiovascular disease. Circulation. 2003; 108(4): 420-425.

36. Mavri A, Alessi MC, Juhan-Vague I. Hypofibrinolysis in the insulin resistance syndrome: implication in cardiovascular diseases. Journal of Internal Medicine. 2004;255(4): 448-456.

37. Juhan-VagueI, Alessi MC, Mavri A, Morange PE. Plasminogen activator inhibitor-1, inflammation, obesity, insulin resistance and vascular risk. Journal of Thrombosis and Haemostasis. 2003; 1: 1575-1579.

38. Dellas C, Loskutoff DJ. Historical analysis of PAI-1 from its discovery to its potential role in cell motility and disease. Thrombosis and Haemostasis. 2005; 934: 631-640.

39. Alessi MC, Juhan-Vague I. PAI-1 and the metabolic syndrome: links, causes, and consequences. Arteriosclerosis, Thrombosis, and Vascular Biology. 2006; 26(10): 2200-2207.

40. Kannel W, Wolf P, Castelli W, D'Agostino R. Fibrinogen and risk of cardiovascular disease: The Framingham Study. The Journal of the American Medical Association. 1987; 258: 1183-1186.

41. Ford ES. The metabolic syndrome and C-reactive protein, fibrinogen, and leukocyte count: findings from the Third National Health and Nutrition Examination Survey. Atherosclerosis. 2003; 168(2): 351-358.

42. Maury E, Noël L, Detry R, Brichard SM. In vitro hyperresponsiveness to tumor necrosis factor-alpha contributes to adipokine dysregulation in omental adipocytes ofobese subjects. The Journal of Clinical Endocrinology and Metabolism. 2009; 94(4): 1393-1400.

43. Lumeng CN, Bodzin JL, Saltiel AR. Obesity induces a phenotypic switch in adipose tissue macrophage polarization. Journal of Clinical Investigation. 2007; 117.1: 175-184. 44. Esposito K, Pontillo A, Di Palo C, Giugliano G, Masella $\mathrm{M}$ et al. Effect of weight loss and lifestyle changes on vascular inflammatory markers in obese women: a randomized trial. The Journal of the American Medical Association. 2003; 289(14): 1799-1804.

45. Goossens GH, Blaak EE, Theunissen R, Duijvesti- jn AM, Clément K et al. Expression of NLRP3 inflammasome and $\mathrm{T}$ cell population markers in adipose tissue are associated with insulin resistance and impaired glucose metabolism in humans. Mol Immunol. 2012; 50:142-149

46. Tsai S, Clemente-Casares X, Revelo XS, Winer S, Winer DA. Are obesity-related insulin resistance and type 2 diabetes autoimmune diseases? Diabetes. 2015; 64(6):1886-1897.

47. van Exel E, Gussekloo J, de Craen AJ, Frölich M, Bootsma-Van Der Wiel A et al. Low production capacity of interleukin-10 associates with the metabolic syndrome and type 2 diabetes: the Leiden 85-plus study. Diabetes. 2002; 51(4): 1088-1092.

48. Rifai N, Ridker PM. Proposed cardiovascular risk assessment algorithm using high sensitive $\mathrm{C}$ - reactive protein and lipid screening. Clinical Chemistry. 2001; 47: 28-30 PubMed .

49. Muntner P, He J, Chen J, Fonseca V, Whelton PK. Prevalence of nontraditional cardiovascular disease risk factors among persons with impaired fasting glucose, impaired glucose tolerance, diabetes, and the metabolic syndrome: analysis of Third National Health and Nutrition Examination Survey (NHANES III). Annals of Epidemiology. 2004; 14: 686.

50. Maury E, Brichard SM. Adipokine dysregulation, adipose tissue inflammation and metabolic syndrome. Molecular and Cellular Endocrinology. 2010; 314: 1-16.

51. Strissel KJ, Stancheva Z, Miyoshi H, Perfield JW, 2nd, DeFuria J et al. Adipocyte death, adipose tissue remodeling, and obesity complications. Diabetes. 2007; 56(12): 2910-2918.

52. Lawrence T, Gilroy DW. Chronic inflammation: a failure of resolution? International Journal of Experimental Pathology. 2007; 88: 85-94.

53. Bourlier V, Zakaroff-Girard A, Miranville A, De Barros S, Maumus $\mathrm{M}$ et al. Remodeling phenotype of human subcutaneous adipose tissue macrophages. Circulation. 2008; 117(6): 806-815.

54. Ditschuneit HH, Flechtner-Mors M, Adler G. Fibrinogen in obesity before and after weight reduction. Obesity Research. 1995; 3(1): 43-48.

55. Sobel BE, Taatjes DJ, Schneider DJ. Intramural plasminogen activator inhibitor type-1 and coronary atherosclerosis. Arteriosclerosis, Thrombosis and Vascular Biology. 2003; 23(11): 1979-1989.

56. Folsom AR, Kaye SA, Sellers TA, Hong CP, Cerhan 
JR et al. Body fat distribution and 5-year risk of death in older women. The Journal of the American Medical Association. 1993; 269(4): 483-487.

57. Rega G, Kaun C, Weiss TW, Demyanets S, Zorn G PubMed et al. Inflammatory cytokines interleukin-6 and oncostatin $\mathrm{m}$ induce plasminogen activator inhibitor- 1 in human adipose tissue. Circulation. 2005; 111(15): 1938-1945. 